\title{
Operando surface chemistry of micro- and nanocubic copper catalysts for electrochemical $\mathrm{CO}_{2}$ reduction
}

\author{
Karla Banjac ${ }^{1,2 *}$, Thanh Hai Phan ${ }^{1,2}$, Fernando P. Cometto ${ }^{1}$, \\ Patrick Alexa ${ }^{3}$, Yunchang Liang ${ }^{1,2}$, Rico Gutzler ${ }^{3}$, and Magalí \\ Lingenfelder ${ }^{1,2 *}$.
}

\footnotetext{
${ }^{1}$ Max Planck-EPFL Laboratory for Molecular Nanoscience and Technology, Ecole Polytechnique Fédérale de Lausanne (EPFL), 1015 Lausanne, Switzerland.

${ }^{2}$ Institut of Physics (IPHYS), Ecole Polytechnique Fédérale de Lausanne (EPFL), 1015 Lausanne, Switzerland.

${ }^{3}$ Max Planck Institute for Solid State Research, 70569 Stuttgart, Germany.

e-mails: karla.banjac@epfl.ch, magali.lingenfelder@epfl.ch
} 


\section{Abstract}

The electrochemical reduction of $\mathrm{CO}_{2}\left(\mathrm{CO}_{2} \mathrm{RR}\right)$ into multicarbon compounds is a promising pathway towards renewable chemicals. Structure-product selectivity studies highlight that copper (100) facets favour $\mathrm{C}_{2+}$ product formation. However, the atomic processes leading to the formation of (100)-rich $\mathrm{Cu}$ cubes remains elusive. Herein, we use $\mathrm{Cu}$ and graphene-protected $\mathrm{Cu}$ surfaces to reveal the differences in structure and composition of common Cu-based electrocatalysts, from nano to micrometer scales. We show that stripping/electrodeposition cycles lead to thermodynamicallycontrolled growth of $\mathrm{Cu}_{2} \mathrm{O}$ micro/nanocubes, while multi-layered $\mathrm{Cu}$ nanocuboids form universally during $\mathrm{CO}_{2} \mathrm{RR}$ upon polarization-driven re-organization of $\mathrm{Cu}^{0}$ atoms. A synergy of electrochemical characterization by scanning tunnelling microscopy (EC-STM), operando EC-Raman and quasioperando X-Ray Photoemission spectroscopy (XPS) allows us to shed light on the role of oxygen on the dynamic interfacial processes of $\mathrm{Cu}$, and to demonstrate that chloride is not needed for the stabilization of cubic Cu nanostructures. 


\section{Introduction}

The electrochemical reduction of carbon dioxide $\left(\mathrm{CO}_{2} \mathrm{RR}\right)$ holds promise for the production of renewable fuels and chemicals ${ }^{1}$. Extensive material screening conducted over the last three decades reveals that copper is the best suited element capable of converting $\mathrm{CO}_{2}$ to hydrocarbons ${ }^{2-4}$. Single crystal studies established the relationship between the structure and $\mathrm{CO}_{2} \mathrm{RR}$ product selectivity showing that $\mathrm{Cu}(100)$ facets are selective towards ethylene production ${ }^{5}$. Similar product selectivity was found for cube-shaped Cu nanoparticles, i.e., nanocubes with lateral size dimensions between several dozens of nanometres up to several micrometers ${ }^{6}$. The geometry of $\{100\}$ planes and $\{110\}$ edges greatly facilitates $\mathrm{C}-\mathrm{C}$ bond formation towards $\mathrm{C}_{2+}$ products ${ }^{6,7}$. This structure-product selectivity relationship is especially viable for ethylene used as a precursor for plastics and ethylene glycol $^{1}$, both yet to be produced through a carbon-neutral pathway.

One of the main obstacles towards economically feasible $\mathrm{CO}_{2} \mathrm{RR}$ is the poor understanding of the reaction steps and complex processes occurring at the electrochemical interfaces during $\mathrm{CO}_{2} \mathrm{RR}$. In a simple picture, both the electrode and the electrical double layer (EDL) are considered static; however, the latest literature reports that dynamic rearrangements of the electrode surface ${ }^{8}$ and $\mathrm{EDL}^{9,10}$ significantly change local reaction environments ${ }^{11}$. Such surface processes significantly change the initial morphologies of both $\mathrm{Cu}$ surfaces and Cu-based nanoparticles (NP). Operando electrochemical scanning tunnelling microscopy (EC-STM) studies reveal that Cu-based catalysts enrich in $\mathrm{Cu}(100)$ facets (multi-layered nanocuboids or mounds) during $\mathrm{CO}_{2} \mathrm{RR} / \mathrm{HER}$, both in the absence ${ }^{12,14}$ and the presence $e^{12,13,14}$ of $\mathrm{CO}_{2}$. Simultaneously, NPs undergo structural evolutions at the micrometre scale: shape transformations ${ }^{15}$ and degradation through a combination of nanoclustering and coalescence ${ }^{16}$.

Structural evolutions occurring over both atomistic and micrometre scales ${ }^{17}$ underline the importance of tackling the open questions on $\mathrm{Cu}$ electro-catalysis through a combination of surface science techniques. A synergy of electrochemical characterization, operando spectroscopies and 
microscopies thus provides an insight into the complex interfacial processes over different lateral and depth scales, while simultaneously overcoming resolution and detection limits inherent to each individual technique ${ }^{18}$. In-situ characterization is especially important for $\mathrm{Cu}$ catalysts due to their tendency to undergo rapid oxidation. The direct visualization of structural transformations at the atomic scale is possible by using scanning probe microscopies $(\mathrm{SPM})^{19}$. High lateral resolution characteristic for SPM is compromised by the fact that only local reaction environments (surface areas $<5 \mu \mathrm{m}$ at reasonable scan rates) can be imaged. Alternatively, scanning electron microscopy (SEM) and Transmission electron microscopy (TEM) is greatly used for NP characterization, but suffer from beam-induced damage, and NP shape loss due to oxidation processes.

Cu catalysts rich in (100) facets can be prepared upon nanostructuring polycrystalline Cu surfaces: either through in situ emergence of nanocuboids during $\mathrm{CO}_{2} \mathrm{RR}^{12}$ or through oxidative-reductive cycling $^{20,21}$. While nanocuboid formation has recently been reported as the main morphological evolution during $\mathrm{CO}_{2} \mathrm{RR}^{12,22}$, micro/nanocubes prepared upon cycling have been widely employed as $\mathrm{CO}_{2} \mathrm{RR}$ catalysts ${ }^{23,21,24,25}$. The cycling protocols employ chloride-containing electrolytes, assuming that the cube precursor is $\mathrm{CuCl}$. Cubic $\mathrm{CuCl}$, presumed to be formed during the anodic scan, converts to $\mathrm{Cu}_{2} \mathrm{O} \mathrm{NCs}{ }^{20,21}$. This hypothesis on $\mathrm{CuCl}-$ to- $-\mathrm{Cu}_{2} \mathrm{O}$ NC transformation, while being valid for $\mathrm{CuCl} N P$ precipitation in liquid-phase $\mathrm{Cu}_{2} \mathrm{O} \mathrm{NCs}$ synthesis based on a hydrolysis approach ${ }^{26}$, disagrees with the fact that chloride anions enhance anodic Cu dissolution during cycling.

Here, we report on the differences between micro/nanocubes and nanocuboids formed in situ during $\mathrm{CO}_{2} \mathrm{RR}$ upon surface nanostructuring. To gain information on their morphologies and chemical compositions, we use a combination of microscopies (SEM, AFM, EC-STM) with operando EC-Raman (deep-surface sensitive) and X-ray photoelectron (near-surface sensitive) spectroscopy studies. Our results establish that the emergence of nanocuboids during $\mathrm{CO}_{2} \mathrm{RR}$ is a general phenomenon on $\mathrm{Cu}$ catalysts due to the potential-driven re-organization of metallic $\mathrm{Cu}$ atoms. Moreover, we show that the cubic morphology is inherent to cycling protocols that lead to Cu dissolution in the anodic scan 
and subsequent electrodeposition in the cathodic scan. Our findings clarify the role of oxygen for stabilization of the microscopic cubic morphology and discard the hypothesis that chloride is needed as a precursor. This study highlights the importance of combined surface science studies for detangling the complex interfacial processes occurring on $\mathrm{Cu}$ during $\mathrm{CO}_{2} \mathrm{RR}$ and thus, offers a novel outlook for synthesis optimization and stability issues related to $\mathrm{Cu}(100)$-rich catalysts.

\section{Results}

\section{Morphology of nanocuboids versus micro/nanocubes}


a

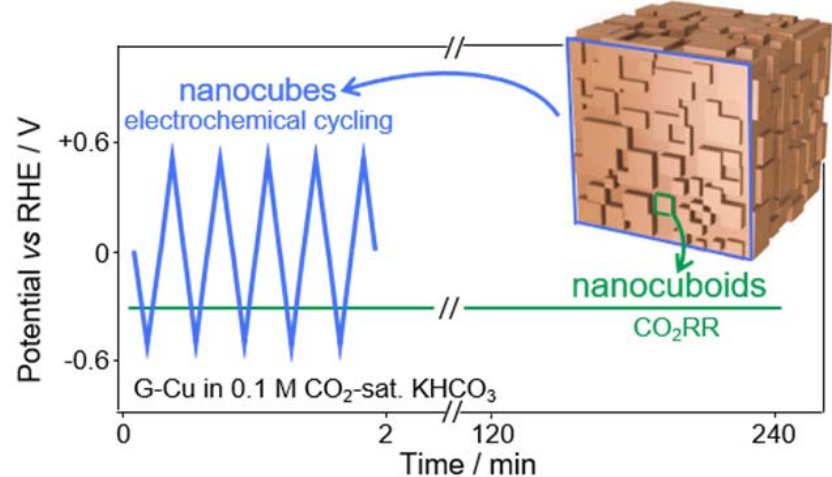

b

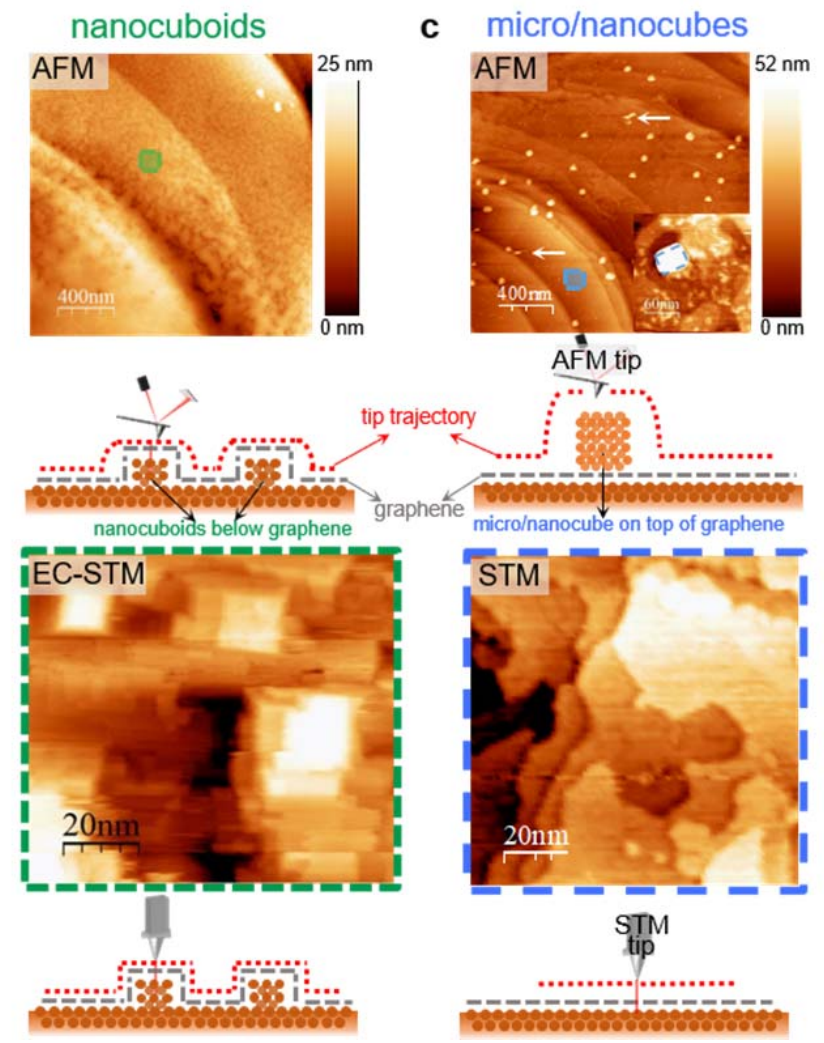

Figure 1. Morphology of Cu-based nanocuboids and micro/nanocubes prepared by electrosynthesis: nanostructuring versus electrochemical cycling. a, Constant-potential polarization of g-Cu at $-1 \mathrm{~V}$ vs Pt pseudo-reference electrode and electrochemical cycling of g-Cu between $-0.6 \mathrm{~V}$ and $+0.6 \mathrm{~V}$ vs RHE in $0.1 \mathrm{M} \mathrm{CO}_{2}$ saturated $\mathrm{KHCO}_{3}$. b, AFM and EC-STM images of the nanocuboids prepared upon surface reconstruction on $\mathrm{g}-\mathrm{Cu}$ together with the corresponding schemes. $\mathbf{c}$, Top panel: AFM height image of $\mathrm{Cu}_{2} \mathrm{O}$ nanocubes formed upon cycling. The white arrows denote the scan lines in which NCs were picked up by the AFM tip. Bottom panel: STM image showing the polycrystalline $\mathrm{Cu}$ underneath $\mathrm{G}$ - after cycling up to $+0.9 \mathrm{~V}$ vs $\mathrm{RHE}$ in $0.1 \mathrm{M} \mathrm{CO}_{2}$ saturated $\mathrm{KHCO}_{3}$. The regions marked on AFM images with the squares are superimposed to highlight the size differences between AFM and STM images.

To study the early stages of the Cu cubic feature formation, we used both polycrystalline pristine $\mathrm{Cu}$ foils ( $\mathrm{Cu}$ ), and $\mathrm{Cu}$ covered by a monolayer of graphene (g-Cu, see Methods for details) that have a 
similar product selectivity for $\mathrm{CO}_{2} \mathrm{RR} .{ }^{12}$ As the role of oxides on $\mathrm{Cu}$ during $\mathrm{CO}_{2} \mathrm{RR}$ is under debate ${ }^{12,27-}$

31, g-Cu was chosen because graphene acts as a protection layer towards air oxidation ${ }^{32}$ allowing us to spectroscopically distinguish the differences in chemical composition between the $\mathrm{Cu}$ micro/nanocubes on top of graphene and the underlying Cu substrate, which remains mostly metallic upon electrochemical/air oxidation. Moreover, its flatness and well-defined electronic structure facilitates high-resolution SPM characterization (Supplementary Fig. 1). In the case of EC-STM, we have recently shown that one can scan preferentially the same region either on top or underneath graphene, by finding the bias conditions where graphene is transparent to STM ${ }^{12}$.

Figure 1 compares the morphologies of the nanocuboids synthetized on $\mathrm{g}$ - $\mathrm{Cu}$ upon constantpotential polarization and the micro/nanocubes prepared upon cycling. Nanocuboids were formed upon constant-potential polarization of $\mathrm{g}-\mathrm{Cu}$ in $0.1 \mathrm{M} \mathrm{CO}_{2}$ saturated $\mathrm{KHCO}_{3}$ (Figure 1.b) ${ }^{12}$. AFM images of as-treated g-Cu show that the initial flat $\mathrm{Cu}$ surface (Supplementary Fig. 1) became 'granular', similar to the uncovered Cu surfaces reported by Arán-Ais et a ${ }^{33}$. High-resolution EC-STM images of the $\mathrm{Cu}$ surface underneath graphene reveal that the nanocuboids are (100) facet multilayers. For g- $\mathrm{Cu}$, graphene remained intact during surface evolution (Supplementary Fig. 2). As a result, nanocuboids were covered by graphene, and no nanoparticles are found on top of graphene (Figure 1.a). 

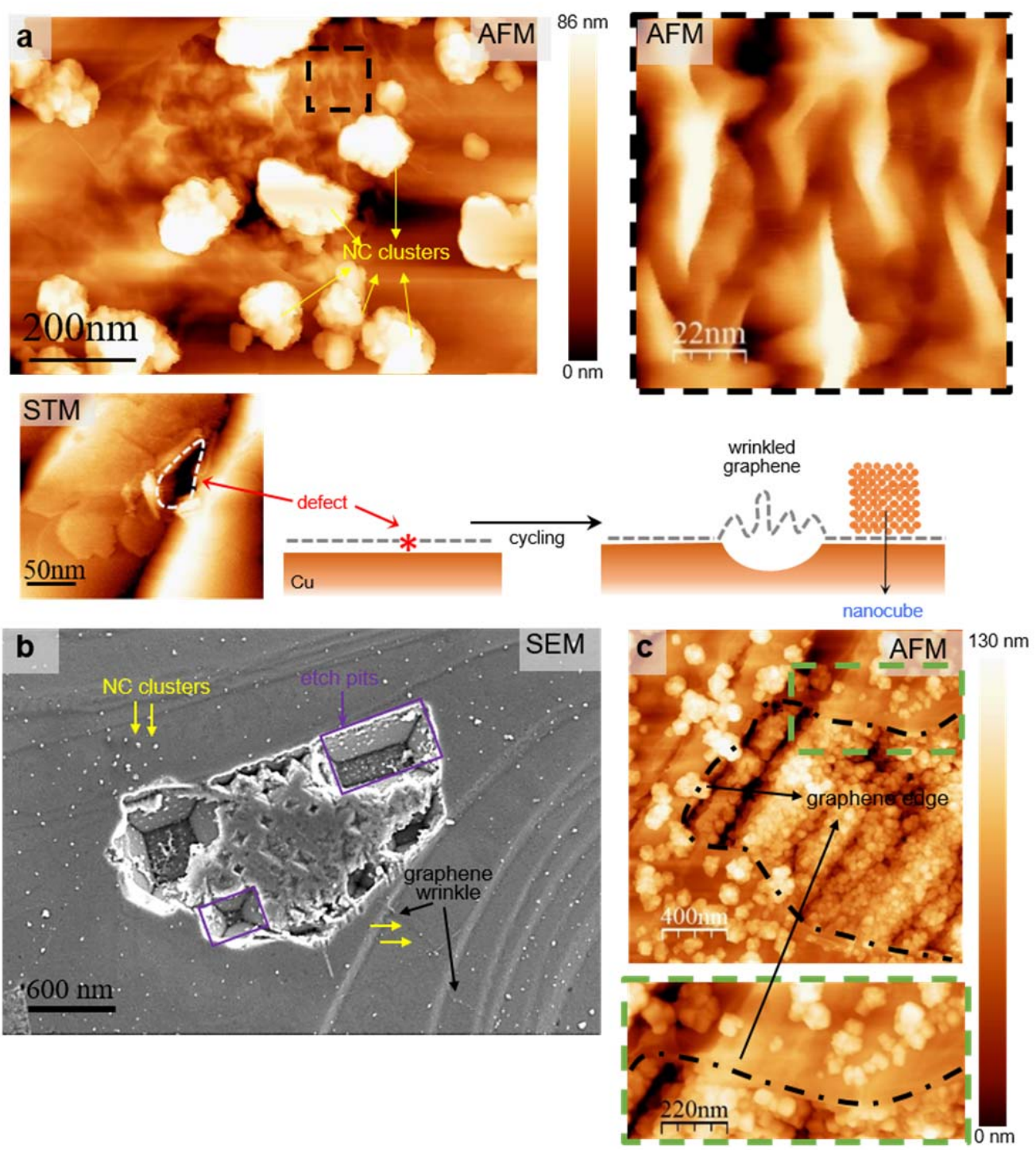

Figure 2. Micro/nanocube clusters prepared upon electrochemical cycling on g-Cu. a, AFM image of micro/nanocube clusters on a wrinkled graphene area together with STM image showing the area of the exposed $\mathrm{Cu}$ surface and unfolded graphene on pristine g-Cu. Graphene wrinkles over the shallow etch pit formed upon local dissolution of the Cu surface (see scheme). b, SEM image showing the micro/nanocube clusters and the etch pits on g-Cu cycled for 101 cycles. c, AFM images showing micro/nanocube formed on top of graphene and close to the graphene defects (here, close to the graphene edge).

When turning to the electrochemical cycling protocol, micro/nanocubes were synthesized by cycling of $\mathrm{g}-\mathrm{Cu}$ in $0.1 \mathrm{M} \mathrm{CO}_{2}$ saturated $\mathrm{KHCO}_{3}$ (Figure 1.c). The protocol was modified from the methods ${ }^{24}$ reported previously in order to prepare small NCs suitable for AFM/STM studies. After five cycles, the surface was sparsely populated with nanocubes (edge length $\approx 25 \mathrm{~nm}^{3}$ ). These nanocubes are on top and weakly attached to graphene; their high mobility thus challenges AFM imaging. STM reveals that 
the Cu surface underneath graphene remains flat and polycrystalline (Supplementary Figs. 3 and 4). Any changes in the cycling protocol affect micro/nanocube size and coverage, both increasing with the number of cycles (Figure 2). Also, the well-defined etch pits on the Cu surface formed after 101 cycles suggest that micro/nanocube formation is closely associated with Cu dissolution of uncovered areas and through graphene defects. Furthermore, the increase in the oxidation vertex potentials to the potentials higher than $+0.6 \mathrm{~V}$ vs RHE resulted in the formation of micro/nanocube clusters rather than stand-alone cubes. The clusters are situated around the areas of wrinkled graphene, likely formed upon relaxation of graphene over the shallow Cu etch pit (Figure 2.b). Additional AFM images of cube clusters confirm that cubes preferentially form close to the graphene defects (Figure 2.c). Finally, no micro/nanocubes were formed when restricting the cycling potential window below the Cu dissolution potential; as in, e.g., an attempt to prepare micro/nanocubes through electrochemical cycling between -0.7 and $-0.2 \mathrm{~V}$ vs RHE (Supplementary Fig. 5). Taken together, these results show that cycling leads to $\mathrm{Cu}$ dissolution in the anodic sweep and electrodeposition of as-dissolved $\mathrm{Cu}$ species on top of graphene in the cathodic sweep.

\section{Chemical composition of cuboids vs micro/nanocubes}

The chemical composition of pristine $\mathrm{Cu}, \mathrm{g}$-Cu before and after in contact with the electrolyte, and nanocuboids and micro/nanocubes prepared upon constant-potential polarization and cycling were studied by quasi-operando XPS (Figure 3.a). The UHV-electrochemistry set-up ${ }^{34}$ allows control over the atmosphere conditions during the sample preparation (conducted inside the UHV-compatible EC cell saturated with $\mathrm{CO}_{2}$ ), direct sample transfer to the UHV environment, and air-free transfer to the XPS set-up.

Cu LMM Auger spectra are used to determine the occurrence of $\mathrm{Cu}^{2+} / \mathrm{Cu}^{+}$species (predominant peaks at $918.4 \mathrm{eV}, 916.5 \mathrm{eV}$, and $917.8 \mathrm{eV}$ are related to metallic $\mathrm{Cu}, \mathrm{Cu}^{+}$and $\mathrm{Cu}^{2+}$, respectively), while the O1s spectra are employed to assess the presence of the oxygen-containing surface species. The O1s peak component centred at $532 \mathrm{eV}$ (marked in grey) remained constant for all treatments; we 
associate it with adsorbed $\mathrm{H}_{2} \mathrm{O} / \mathrm{OH}$ species $^{35}$ and surface adsorbates ${ }^{36}$ (see AFM image in Supplementary Fig. 6) with a minor contribution from graphene defects ${ }^{37,38}$ (consistent with the C1s spectra in Supplementary Fig. 7, showing a dominant $C \mathrm{sp}^{2}$ peak and minor contributions from $\mathrm{C}=0$ and $\mathrm{C}-\mathrm{O})$. On the other hand, the $\mathrm{O} 1 \mathrm{~s}$ peaks at $530.2 \mathrm{eV}$ and $529.7 \mathrm{eV}$ are attributed to the presence of $\mathrm{Cu}_{2} \mathrm{O}$ and $\mathrm{CuO}$, respectively ${ }^{37,39}$.

The pristine g-Cu sample was slightly oxidized with a dominant Cu LMM signal from metallic $\mathrm{Cu}$ and a minor amount of $\mathrm{Cu}_{2} \mathrm{O}$ and $\mathrm{CuO}$ (Figure 3.a, red panel). Both $\mathrm{Cu}_{2} \mathrm{O}$ and $\mathrm{CuO}$ components are due to $\mathrm{Cu}_{x} \mathrm{O}$ formed upon the unavoidable local ambient oxidation of $\mathrm{Cu}$, proceeded by intercalation of oxygen and water through the graphene defects, as suggested by Kwak et $\left.a\right|^{32}$. Furthermore, the CuLMM spectrum of the $\mathrm{g}$ - $\mathrm{Cu}$ after the formation of the nanocuboids during $\mathrm{CO}_{2} \mathrm{RR}$ displays metallic $\mathrm{Cu}$ with a small amount of $\mathrm{Cu}_{2} \mathrm{O}$ (Figure 3.a, green panel). As the initial oxidation state can vary from sample to sample and we cannot fully discard the presence of oxygen traces during the transfer, it is important to point out that XPS/Auger analysis shows only qualitative trends, even when the measurements were performed in the exact same samples before and after the EC treatments to do meaningful comparisons. These results, together with the operando EC-Raman spectra discussed below, indicate that the surface remains metallic during $\mathrm{CO}_{2} \mathrm{RR}$ but $\mathrm{Cu}_{2} \mathrm{O}$ is rapidly formed once the sample is at open circuit potential. In agreement with AFM (Figure 1.b), STM results (Supplementary Fig. 2) and Raman spectra ${ }^{12}$, the $\mathrm{C} 1 \mathrm{~s}$ spectrum obtained on the g-Cu sample with nanocuboids (Supplementary Fig. 7) confirms that the emergence of nanocuboids creates no additional defects in graphene and that they are fully covered by graphene. In contrast, micro/nanocubes prepared upon electrochemical cycling are mostly composed of $\mathrm{Cu}_{x} \mathrm{O}$ (Figure 3.a, blue panel). Deconvolution of CuLMM spectrum reveals that micro/nanocubes on top of $\mathrm{g}-\mathrm{Cu}$ have $38 \% \mathrm{Cu}^{\circ}, 58 \% \mathrm{Cu}_{2} \mathrm{O}$, and $4 \%$ CuO. Therefore, a simultaneous increase in the $\mathrm{CuO}$ and $\mathrm{Cu}_{2} \mathrm{O}$ O1s components after the cycling protocol confirms the formation of $\mathrm{Cu}_{x} \mathrm{O}$ micro/nanocubes. 
a

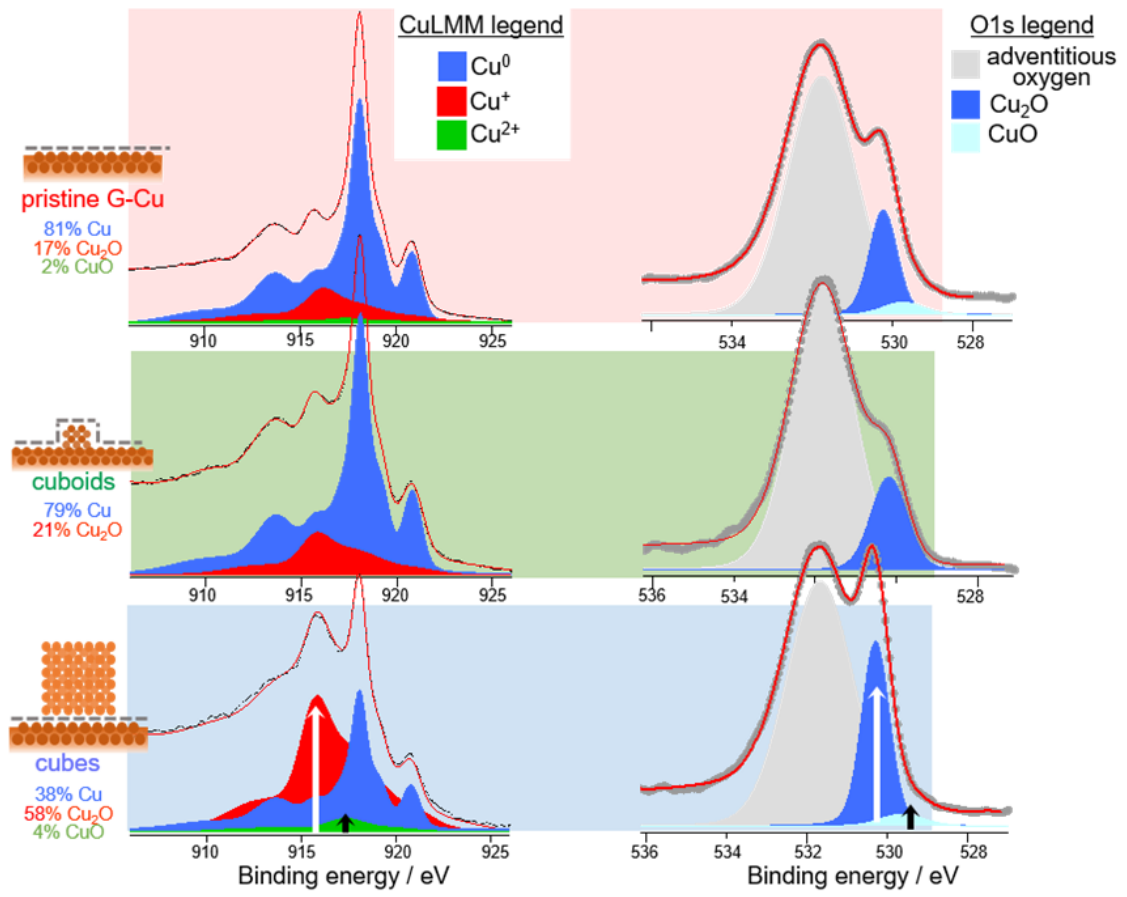

b (i)

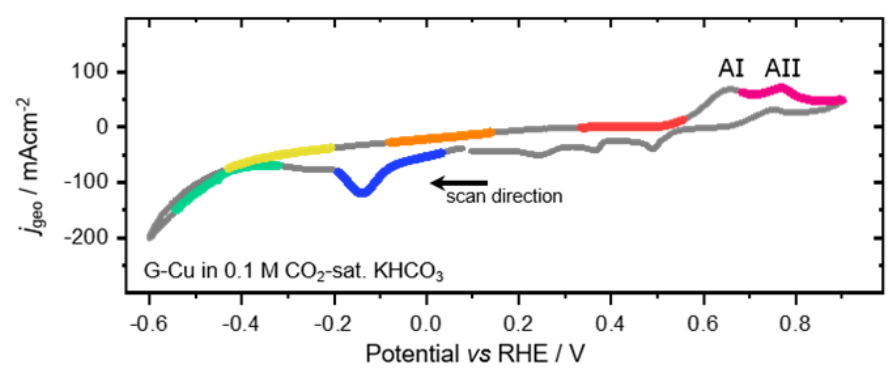

(ii) cathodic scans
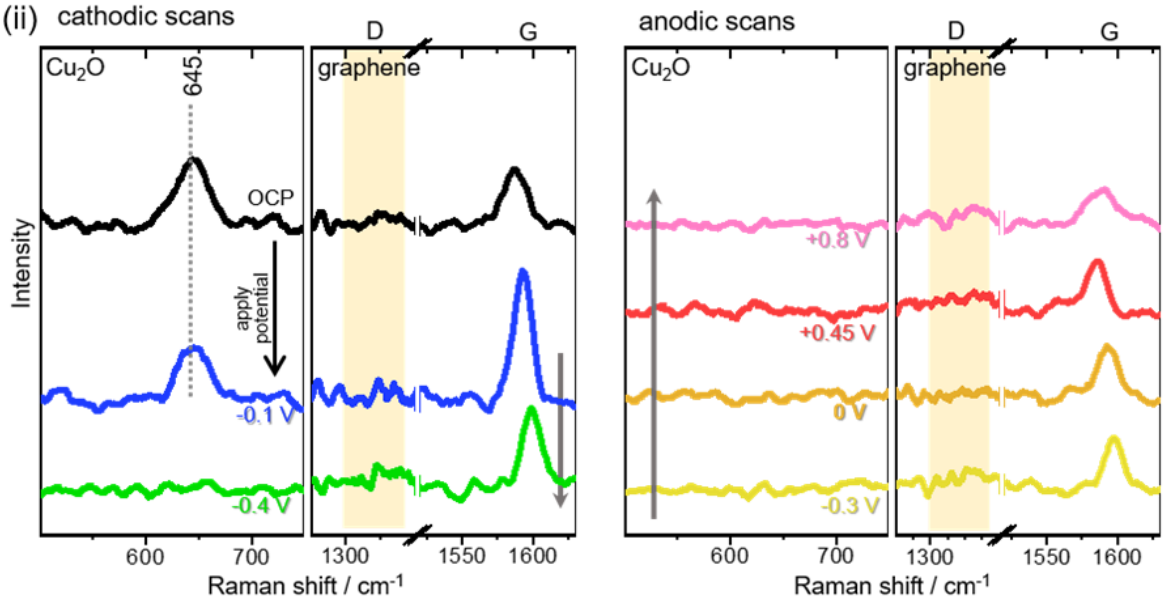

Figure 3 (Quasi-) operando spectroscopy of $\mathrm{Cu}$ nanocubes. a, Quasi-operando XPS spectra of as-received g-Cu, nanocuboids, and nanocubes. b, Operando EC Raman spectroscopy of g-Cu recorded at different potentials during cycling. (i) Cyclic voltammogram of g-Cu in $0.1 \mathrm{M} \mathrm{CO}_{2}$ saturated $\mathrm{KHCO}_{3}(\mathrm{pH}=6.8)$ recorded at a scan rate of $5 \mathrm{mV} / \mathrm{s}$. The arrow denotes the scanning direction. Different colors denote the potential ranges over which operando Raman spectra, in (ii), were recorded. 
As a control experiment aiming to test the role of graphene on the chemical identity of nanocuboids vs micro/nanocubes, we performed the same experiments on polycrystalline Cu foil (Supplementary Fig. 9). The Cu surface remains mostly metallic upon in situ emergence of the nanocuboids during $\mathrm{CO}_{2} \mathrm{RR}$, while similar trends in the increase of the $\mathrm{Cu}_{2} \mathrm{O}$ component in the $01 \mathrm{~s}$ spectra and a decrease in the $\mathrm{Cu} / \mathrm{Cu}^{+}$ratio observed after electrochemical cycling confirm differences between the chemical composition of nanocuboids vs micro/nanocubes.

We remark that quasi-operando XPS measurements were conducted under open circuit potential (OCP) conditions, i.e. rapid local oxidation through the graphene defects occurs. To gain further insights into the micro/nanocube formation during cycling, we tracked the changes in the chemical state of g-Cu during the first cycle using operando Raman spectroscopy. Characteristic Raman signatures of $\mathrm{Cu}_{x} \mathrm{O}$ and graphene allow us to investigate simultaneously the oxidation state of $\mathrm{Cu}$ and the stability of graphene. Raman spectra were recorded at different potentials as g-Cu electrode was cycled in $0.1 \mathrm{M} \mathrm{CO}_{2}$ saturated $\mathrm{KHCO}_{3}$ between $-0.6 \mathrm{~V}$ and $+0.9 \mathrm{~V}$ vs RHE. The spectra acquired at OCP reveal the presence of the $\mathrm{Cu}_{2} \mathrm{O}$ band $\left(645 \mathrm{~cm}^{-1}\right)^{40,41}$ and the characteristic graphene $\mathrm{G}$ band (1585 $\left.\mathrm{cm}^{-1}\right)^{41}$. This result, together with quasi-operando XPS results for the g-Cu before and after the electrolyte contact (Supplementary Fig. 8-9), suggests that pristine g-Cu is slightly oxidized due to air oxidation. The reduction of the native oxide starts at the potential of $\approx 0 \mathrm{~V} v s \mathrm{RHE}$, while it gets fully reduced at potentials less negative than $-0.2 \mathrm{~V}$ vs RHE. In the anodic scan, $\mathrm{Cu}$ under graphene remains metallic for potentials up to $+0.9 \mathrm{~V}$ vs RHE. Simultaneous appearance of the $\mathrm{Cu}$ oxidation peaks (denoted as $\mathrm{Al}$ and $\mathrm{All}$ in Figure 3.b and assigned to oxidation) suggests oxidation of Cu surface exposed through graphene defects. We also tested the oxidation behaviour of g-Cu over the wide potential range (Supplementary Fig. 10). The $\mathrm{Cu}$ surface under high-quality graphene remains metallic after the potential sweeps up to $+1.1 \mathrm{~V} v s$ RHE.

\section{Effect of chloride on the Cu micro/nanocube morphology}


To investigate the role of additives on the $\mathrm{Cu}_{2} \mathrm{O}$ micro/nanocube growth, we examined the effect of chloride. $\mathrm{Cu}_{x} \mathrm{O}$ micro/nanocubes were prepared upon electrochemical cycling between $-0.6 \mathrm{~V}$ and $+0.9 \mathrm{~V}$ vs RHE in chloride-free $0.1 \mathrm{M} \mathrm{CO}_{2}$ saturated $\mathrm{KHCO}_{3}$ (Figure $4 . \mathrm{a}$ ) and in $4 \mathrm{mM} \mathrm{KCl}+0.1 \mathrm{M} \mathrm{CO}_{2}$ saturated $\mathrm{KHCO}_{3}$ (Figure 4.b). SEM was used to characterize the micro/nanocubes prepared on polycrystalline $\mathrm{Cu}$ foil after 100 cycles, AFM was used to visualize the micro/nanocubes prepared on g-Cu after five cycles. Cubic contours are discernable on both $\mathrm{Cu}$ foil and g-Cu cycled in the chloridefree electrolyte. On g-Cu, AFM reveals the smooth morphology of the underlying $\mathrm{g}$ - $\mathrm{Cu}$ and micro/nanocube clusters randomly distributed along the g-Cu substrate. 

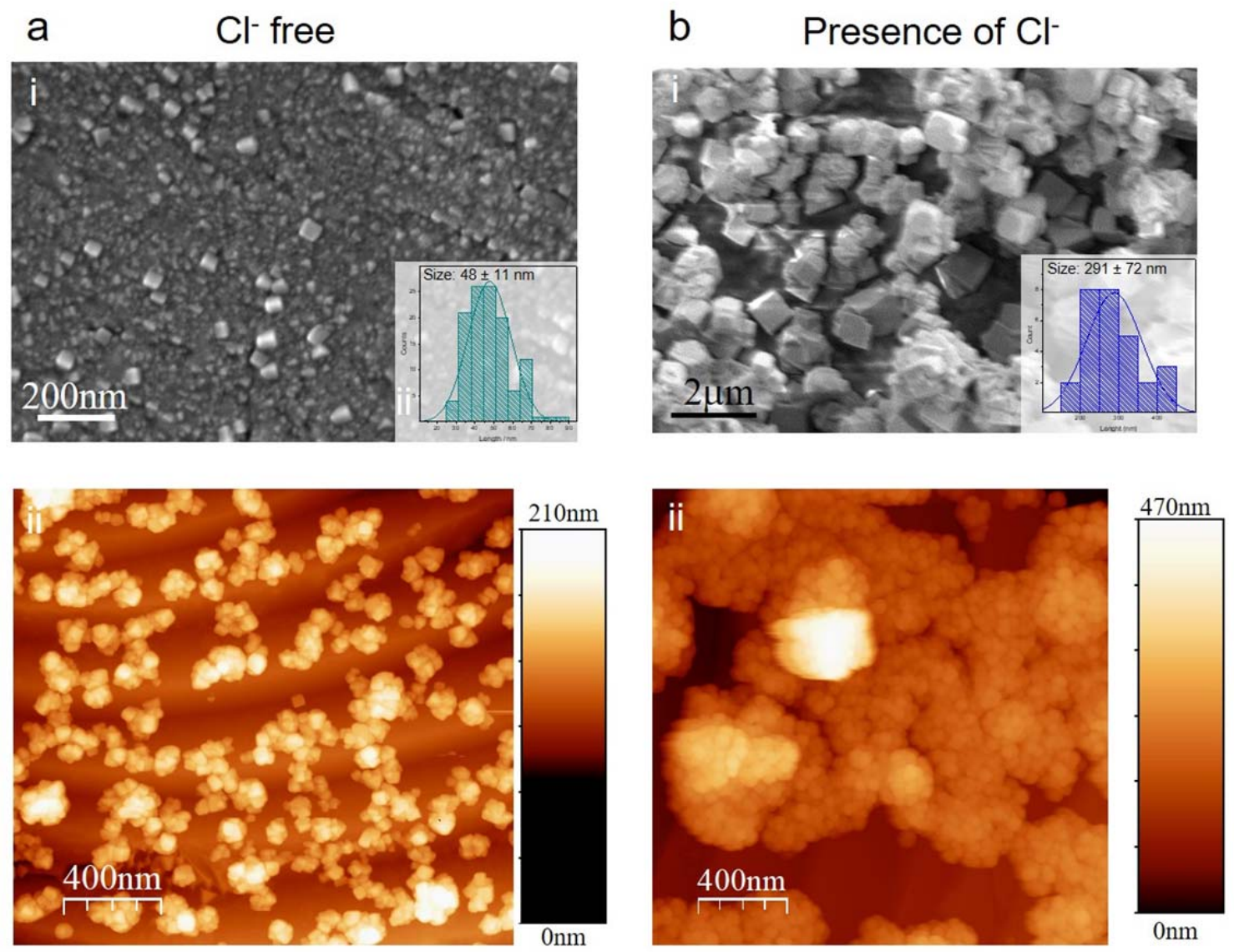

Figure 4. Effect of chloride on the $\mathrm{Cu}_{x} \mathrm{O}$ micro/nanocube formation. a, $\mathrm{Cu}$ micro/nanocubes synthesized in a chloride-free electrolyte. (i) SEM image of $\mathrm{Cu}_{x} \mathrm{O}$ micro/nanocubes prepared upon cycling of a polycrystalline $\mathrm{Cu}$ foil in a chloride-free electrolyte together with the corresponding size distribution. (ii) AFM image of $\mathrm{Cu}$ micro/nanocubes on graphene upon cycling of $\mathbf{g}-\mathrm{Cu} . \mathbf{b}, \mathrm{Cu}_{x} \mathrm{O}$ micro/nanocubes synthesized in a chloride-containing electrolyte. (i) SEM image of the $\mathrm{Cu}_{x} \mathrm{O}$ micro/nanocubes prepared upon electrochemical cycling of polycrystalline $\mathrm{Cu}$ foil in a chloride-containing electrolyte together with the corresponding size distribution. (ii) AFM image of $\mathrm{Cu}_{x} \mathrm{O}$ micro/nanocubes on g-Cu synthesized upon electrochemical cycling.

The stripping/redeposition cycling method gradually stabilizes a cubic morphology and selectively exposes the (100) facets of $\mathrm{Cu}_{2} \mathrm{O}^{24,42}$. NP prepared in chloride-containing electrolytes exhibit similar structures as the ones prepared without chloride, but are six times larger. The most likely mechanism involves chloride enhancing the local dissolution of $\mathrm{Cu}$, leading to the high concentration of the $\mathrm{Cu}$ ions close to the surface, which get electrodeposited in the cathodic scan on the graphene.

\section{Discussion}


The combination of electron and scanning probe microscopies characterization with operando spectroscopies highlights the morphological and compositional differences between Cu nanocuboids formed during $\mathrm{CO}_{2} \mathrm{RR}$ and $\mathrm{Cu}_{x} \mathrm{O}$ micro/nanocubes prepared upon electrochemical cycling. Moreover, we employ g-Cu as a model system that allows us to distinguish the differences in surface processes leading to the formation of metallic Cu nanocuboids underneath graphene vs the ones resulting in $\mathrm{Cu}_{2} \mathrm{O}$ micro/nanocube formation on top of graphene. This approach gives an unprecedented insight into their formation mechanisms.

Cu-based $\mathrm{CO}_{2} \mathrm{RR}$ catalysts undergo nanostructuration during $\mathrm{CO}_{2} \mathrm{RR}$ (Figure 5, top panel). We highlight that nanocuboids are (100) facet multilayers (mounds) with lateral dimensions less than 100 $\mathrm{nm}$. Their heights are determined by the number of the surface layers per mound, strongly dependant on the conditions of the surface prior to $\mathrm{CO}_{2} \mathrm{RR}$ and $\mathrm{CO}_{2} \mathrm{RR}$ conditions (electrolyte, potential, polarization time). Nanocuboids are imperceptible by $\mathrm{SEM}^{43}$. Moreover, they lose their square contours upon ambient oxidation and thus, appear as granular structures in post-mortem AFM and STM studies. This highlights the importance of operando surface-sensitive studies for understanding the in situ surface physics processes during $\mathrm{CO}_{2} \mathrm{RR}$, including nanocuboid formation as a morphological evolution occurring on all $\mathrm{Cu}$ electrocatalysts during $\mathrm{CO}_{2} \mathrm{RR}$, regardless of their microscopic precursor morphology ${ }^{12,22}$. 

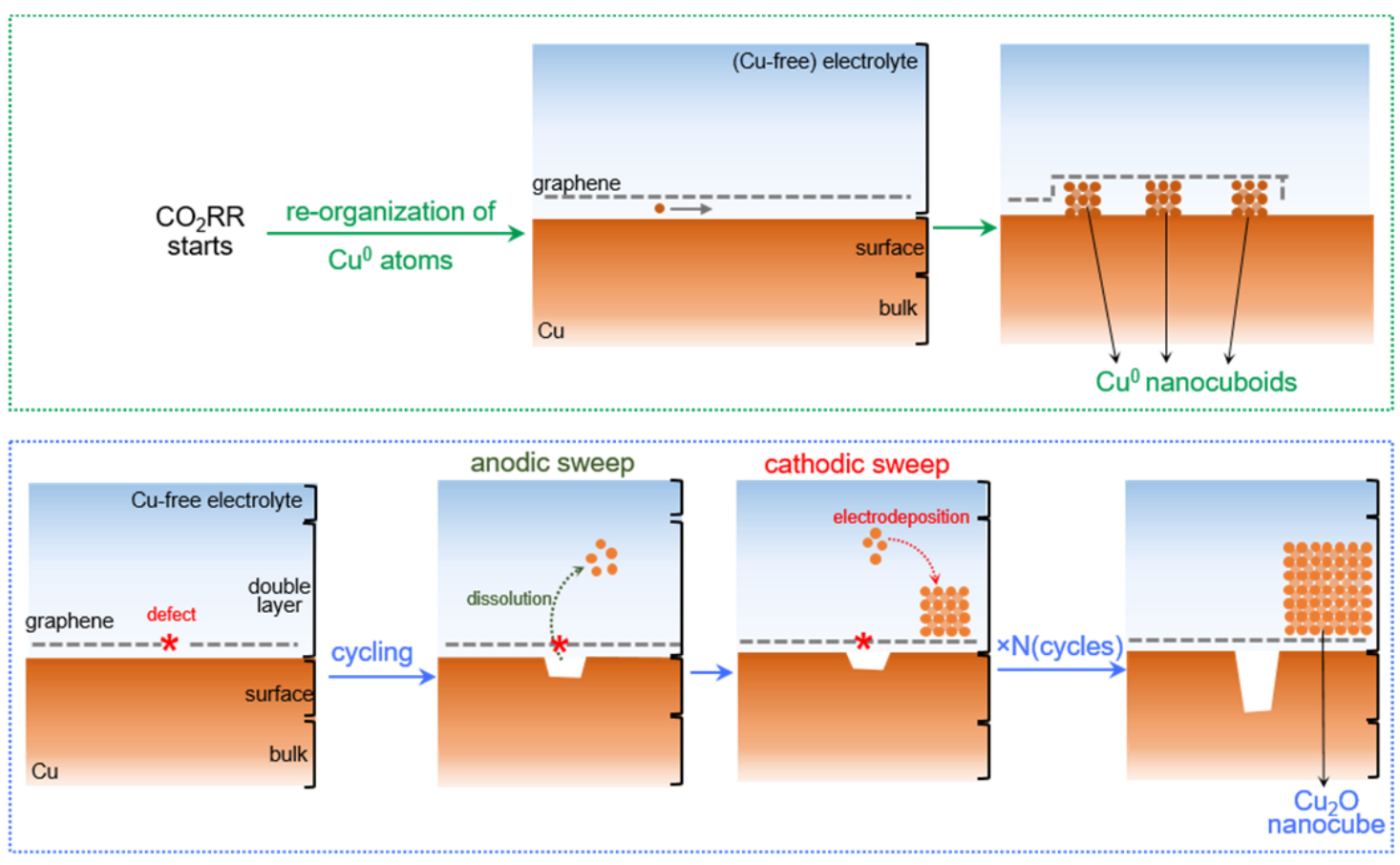

Figure 5. Formation mechanisms of the nanocuboids and micro/nanocubes. Top panel: Scheme showing the formation of the $\mathrm{Cu}$ nanocuboids upon potential-driven re-organization of the metallic $\mathrm{Cu}$ atoms during $\mathrm{CO}_{2} \mathrm{RR}$. Bottom panel: Scheme showing the formation of $\mathrm{Cu}_{2} \mathrm{O}$ micro/nanocubes through sequential dissolution/electrodeposition cycles.

EC-STM, quasi-operando XPS (near-surface sensitive) and operando EC-Raman spectroscopy (deepsurface sensitive) confirm that the nanocuboids are metallic $\mathrm{Cu}(100)$ mounds. The native oxide, if present, is fully reduced at potentials more negative than $0 \mathrm{~V} v$ s $\mathrm{RHE}$, i.e., at low $\mathrm{CO}_{2} \mathrm{RR}$ potentials ${ }^{27,31}$. Furthermore, our spectroscopic data allow us to discard the role of $\mathrm{Cu}^{+}$species on the nanocuboid formation that occurs via the re-organization of metallic $\mathrm{Cu}$ atoms in the top-most surface layers (Figure 5, top panel). ${ }^{12}$

When turning to the electrochemical cycling protocol, we demonstrate that the preparation of $\mathrm{Cu}_{2} \mathrm{O}$ micro/nanocubes occurs through electrochemical cycling both in chloride-free and chloridecontaining electrolytes. The observation of micro/nanocube formation in chloride-free electrolytes, together with the absence of $\mathrm{CuCl}$ intermediates upon cycling reported by Eilert et al. in chloridecontaining electrolytes ${ }^{44}$, disproves the hypothesis that $\mathrm{CuCl}$ determines the cubic morphology of the as-formed micro/nanocubes ${ }^{20,21}$. 
From the above results, we propose the formation mechanism of micro/nanocubes upon electrochemical cycling of $\mathrm{Cu}$ surfaces in $\mathrm{Cu}$-ion free electrolytes (Figure 5, bottom panel). In the anodic sweep, the Cu surface exposed to the electrolyte through graphene defects dissolves, while $\mathrm{Cu}$ surface underneath graphene remains metallic. This suggests that the exposed $\mathrm{Cu}$ areas act as local $\mathrm{Cu}$ ions sources, while both graphene and $\mathrm{Cu}$ underneath it are electrochemically inactive. These $\mathrm{Cu}$ ions in the solution then get reduced and electrodeposited on top of graphene in the cathodic sweep. As-formed nuclei transform into the $\mathrm{Cu}_{2} \mathrm{O}$ micro/nanocubes in the consecutive stripping/deposition cycles ${ }^{46}$. Cube morphology is thus a direct consequence of the thermodynamic stability of the $\mathrm{Cu}_{2} \mathrm{O}(100)$ facets, where the surface energies of $\mathrm{Cu}_{2} \mathrm{O}$ increase in the following order: $\gamma(100)<\gamma(111)<\gamma(110)^{47}$

Cu dissolution at such low potentials is rather surprising. One should bear in mind that corresponding CVs appear featureless, which might be misinterpreted as if neither surface morphology nor the chemical state is changing. However, the corrosive properties of $\mathrm{KHCO}_{3}$ electrolyte have been suggested earlier ${ }^{48}$, and only a recent study shows that dissolution to $\mathrm{Cu}^{+}$starts at $+0.5 \mathrm{~V}$ vs $\mathrm{RHE}^{45}$. Interestingly, dissolution occurs regardless of the local $\mathrm{pH}$.

This mechanism also describes well $\mathrm{Cu}_{2} \mathrm{O}$ micro/nanocube formation on a $\mathrm{Cu}$ foil. Our results suggest that the stabilization of $\mathrm{Cu}_{2} \mathrm{O}(100)$ facets upon dissolution/electrodeposition cycles seems more likely than the direct oxidation to $\mathrm{Cu}(\mathrm{II})$ carbonate-hydroxide micro/nanocubes, which occurs upon prolonged polarization at $+0.7 \mathrm{~V}$ vs $\mathrm{RHE}^{44}$, rather than upon cycling. The same mechanism also explains the formation of $\mathrm{Cu}_{x} \mathrm{O}$ NCs prepared upon cycling to high oxidation potentials (e.g., $>+0.8 \mathrm{~V}$ vs RHE in reference ${ }^{45}$ and ${ }^{49}$ ), where $\mathrm{Cu}$ dissolution to $\mathrm{Cu}^{2+}$ ions leads to the electrodeposition in the cathodic sweeps.

Our observations rule out the need of chloride in the $\mathrm{Cu}_{x} \mathrm{O}$ micro/nanocube formation. Chloride, often added as an additive in electrochemical cycling ${ }^{25}$, changes the oxidation mechanism and enhances the local dissolution ${ }^{50}$. This strategy allows control over the $\mathrm{Cu}_{x} \mathrm{O}$ (micro/nano)cube size 
and coverage: high concentration of $\mathrm{Cu}$ species, here formed upon anodic dissolution of $\mathrm{Cu}$ surface, leads to the formation of larger cubes densely populating the substrate. However, the cubic morphology is inherent to the cycling protocols.

\section{Conclusions}

In summary, we have revealed the mechanisms of formation of $\mathrm{Cu}_{2} \mathrm{O}$ micro/nanocubes and $\mathrm{Cu}(100)$ nanocuboids through two distinct in-situ electrosynthesis methods, namely electrochemical cycling and potential driven nanostructuration. Electrosynthesis protocols relying on cycling originate from the stripping/electrodeposition cycles, which lead to the growth of the $\mathrm{Cu}_{2} \mathrm{O}$ nanocrystals and their evolution to micro/nanocubes. The cube morphology is a result of the thermodynamics of the crystal $\mathrm{Cu}_{2} \mathrm{O}$ growth in the absence of any additives. Chloride anions, often added during electrosynthesis, enhance $\mathrm{Cu}$ dissolution in the stripping cycle, but have no role in the stabilization of the NCs. Regardless of the inital micro/nanocubic structure, under $\mathrm{CO}_{2} \mathrm{RR}$ conditions, the oxide layer is removed and a polarization-driven nanostructuration of the surface takes place, resulting in the reorganization of the metallic $\mathrm{Cu}$ atoms into few nanometer-sized metallic nanocuboids. Our results highlight that a combination of the complex surface processes and electrochemical reactions occurring in the presumably safe electrochemical windows drastically change the morphologies of Cu-based catalysts. These morphological changes are imperceptible for both post-mortem and surface-insensitive spectroscopic studies, but are key to explain the trends observed in $\mathrm{CO}_{2} \mathrm{RR}$ product selectivity that are ultimately the result of processes occurring at the atomic scale. 


\section{Methods}

Samples. All experiments were performed on bare, polycrystalline $\mathrm{Cu}$ foils or on graphene-covered polycrystalline $\mathrm{Cu}$ foils ( $\mathrm{g}-\mathrm{Cu}$ ). More details can be found in Supplementary Information.

Sample preparation: nanocuboids. $\mathrm{Cu}$ nanocuboids form during $\mathrm{CO}_{2} \mathrm{RR}$ : the samples with $\mathrm{Cu}$ nanocuboids were thus prepared following the reference 12, i.e., upon chronoamperometric polarization at $-1 \mathrm{~V}$ vs Pt pseudo-reference electrode (-0.03 vs $\mathrm{RHE})$ in $0.1 \mathrm{M} \mathrm{CO}_{2}$ sat. $\mathrm{KHCO}_{3}$ for 4 hours.

Sample preparation: micro/nanocubes. $\mathrm{Cu}_{2} \mathrm{O}$ micro/nanocubes were prepared on g- $\mathrm{Cu}$ and bare $\mathrm{Cu}$ foils upon electrochemical cycling in $0.1 \mathrm{M} \mathrm{CO}_{2}$ sat. $\mathrm{KHCO}_{3}$ or $4 \mathrm{mM} \mathrm{KCl} / 0.1 \mathrm{M} \mathrm{CO}_{2}$ sat. $\mathrm{KHCO}_{3}$ in a potential window between $-0.6 \mathrm{~V}$ and $+0.6 \mathrm{~V}$ vs RHE or up to $+0.9 \mathrm{~V}$ vs RHE at a sweep rate of $50 \mathrm{mV} / \mathrm{s}$ for five, twenty or 101 cycles.

Morphological characterization. SEM images were acquired on Zeiss Gemini SEM using an accelerating voltage of $3 \mathrm{kV}$. AFM images were acquired in Peak Force mapping mode on Fast Scan Bio AFM (Bruker) coupled with Nanoscope Controller $V$ and Nanoscope Software 8.15. We use FastScan-B tips (Bruker, radius of $5 \mathrm{~nm}$, nominal $k$ values of $1.8 \mathrm{~N} / \mathrm{m}$ ). STM characterization was carried out on the Dimension Icon STM (Bruker) coupled with the Nanoscope Controller $\mathrm{V}$ and Nanoscope Software 8.15 in a constant-current mode. STM and EC-STM characterization was carried out on freshly-prepared samples at room temperature.

Quasi-operando XPS. To minimize oxidation of the sample after electrochemical treatments, the samples characterized by XPS were prepared in the controlled environment and transferred to XPS chamber through an air-free transfer system. A home-built sample transfer system between UHV and electrochemical environment was implemented ${ }^{34}$. The samples were then transferred to the XPS using a vacuum suitcase (Ferrovac). The XPS measurement was conducted on a commercial Kratos AXIS Ultra system with a monochromatized AI K $\alpha$ source with a base pressure in the lower range. The fitting procedure is described in the Supplementary information.

\section{Raman spectroscopy.}

Operando Raman studies were performed on an inVia Raman spectrometer (Renishaw) coupled with a confocal microscope, VersaSTAT 4 Potentiostat (Ametek), and a home-built electrochemical cell made of polytetrafluoroethylene. Raman spectra were collected using a water-immersion objective (Leica, 64×), $488 \mathrm{~nm}$ laser in line illumination mode, and 2400 lines $/ \mathrm{mm}$ gratings. Line length was $\approx$ $12 \mu \mathrm{m}$; eight spectra were collected over $74.4 \mu \mathrm{m}^{2}$ area. Exposure time was 35 seconds per four spectra. The cell was equipped with a silver/silver chloride $(\mathrm{Ag} / \mathrm{AgCl})$ reference electrode immersed in $3 \mathrm{M} \mathrm{NaCl}$ solution (ProSense) and a coiled gold wire as a counter electrode. Cyclic voltammetry and linear sweep voltammetry were carried out on $\mathrm{g}$ - $\mathrm{Cu}$ samples as working electrodes $\left(A_{\text {geo }} \approx 0.28 \mathrm{~cm}^{2}\right)$. The cell was equipped with a silver/silver chloride $(\mathrm{Ag} / \mathrm{AgCl})$ reference electrode immersed in $3 \mathrm{M}$ $\mathrm{NaCl}$ solution (ProSense) and a coiled gold wire as a counter electrode. 


\section{References}

1. Nitopi, S. et al. Progress and Perspectives of Electrochemical CO2 Reduction on Copper in Aqueous Electrolyte. Chem. Rev. 119, 7610-7672 (2019).

2. Peterson, A. A., Abild-Pedersen, F., Studt, F., Rossmeisl, J. \& Nørskov, J. K. How copper catalyzes the electroreduction of carbon dioxide into hydrocarbon fuels. Energy Environ. Sci. 3, 1311-1315 (2010).

3. Zhou, Y. et al. Dopant-induced electron localization drives $\mathrm{CO} 2$ reduction to $\mathrm{C} 2$ hydrocarbons. Nat. Chem. 10, 974-980 (2018).

4. Jiao, J. et al. Copper atom-pair catalyst anchored on alloy nanowires for selective and efficient electrochemical reduction of CO2. Nat. Chem. 11, 222-228 (2019).

5. Hori, Y., Takahashi, I., Koga, O. \& Hoshi, N. Selective Formation of C2 Compounds from Electrochemical Reduction of $\mathrm{CO} 2$ at a Series of Copper Single Crystal Electrodes. J. Phys. Chem. B 106, 15-17 (2002).

6. Loiudice, A. et al. Tailoring Copper Nanocrystals towards C2 Products in Electrochemical CO2 Reduction. Angew. Chem. Int. Ed. 55, 5789-5792 (2016).

7. Mangione, G., Huang, J., Buonsanti, R. \& Corminboeuf, C. Dual-Facet Mechanism in Copper Nanocubes for Electrochemical CO2 Reduction into Ethylene. J. Phys. Chem. Lett. 10, 4259-4265 (2019).

8. Auer, A. et al. Self-activation of copper electrodes during $\mathrm{CO}$ electro-oxidation in alkaline electrolyte. Nat. Catal. 3, 797-803 (2020).

9. Ledezma-Yanez, I. et al. Interfacial water reorganization as a pH-dependent descriptor of the hydrogen evolution rate on platinum electrodes. Nat. Energy 2, 1-7 (2017).

10. Strmcnik, D. et al. The role of non-covalent interactions in electrocatalytic fuel-cell reactions on platinum. Nat. Chem. 1, 466-472 (2009).

11. Magnussen, O. M. \& Groß, A. Toward an Atomic-Scale Understanding of Electrochemical Interface Structure and Dynamics. J. Am. Chem. Soc. 141, 4777-4790 (2019). 
12. Phan, T. H. et al. Emergence of Potential-Controlled Cu-Nanocuboids and Graphene-Covered Cu-Nanocuboids under Operando CO2 Electroreduction. Nano Lett. 21, 2059-2065 (2021).

13. Kim, Y.-G., Baricuatro, J. H., Javier, A., Gregoire, J. M. \& Soriaga, M. P. The evolution of the polycrystalline copper surface, first to $\mathrm{Cu}(111)$ and then to $\mathrm{Cu}(100)$, at a fixed $\mathrm{CO}_{2} \mathrm{RR}$ potential: a study by operando EC-STM. Langmuir 30, 15053-15056 (2014).

14. Kim, Y.-G., Baricuatro, J. H. \& Soriaga, M. P. Surface Reconstruction of Polycrystalline Cu Electrodes in Aqueous KHCO3 Electrolyte at Potentials in the Early Stages of $\mathrm{CO} 2$ Reduction. Electrocatalysis 9, 526-530 (2018).

15. Kim, D., Kley, C. S., Li, Y. \& Yang, P. Copper nanoparticle ensembles for selective electroreduction of CO2 to C2-C3 products. Proc. Natl. Acad. Sci. 114, 10560-10565 (2017).

16. Huang, J. et al. Potential-induced nanoclustering of metallic catalysts during electrochemical CO 2 reduction. Nat. Commun. 9, 3117 (2018).

17. Birdja, Y. Y. et al. Advances and challenges in understanding the electrocatalytic conversion of carbon dioxide to fuels. Nat. Energy 4, 732-745 (2019).

18. Handoko, A. D., Wei, F., Jenndy, Yeo, B. S. \& Seh, Z. W. Understanding heterogeneous electrocatalytic carbon dioxide reduction through operando techniques. Nat. Catal. 1, 922 (2018).

19. Liang, Y. et al. Electrochemical Scanning Probe Microscopies in Electrocatalysis. Small Methods 3, 1800387 (2019).

20. Roberts, F. S., Kuhl, K. P. \& Nilsson, A. High selectivity for ethylene from carbon dioxide reduction over copper nanocube electrocatalysts. Angew. Chem. Int. Ed Engl. 54, 5179-5182 (2015).

21. Kwon, Y., Lum, Y., Clark, E. L., Ager, J. W. \& Bell, A. T. $\mathrm{CO}_{2}$ Electroreduction with Enhanced Ethylene and Ethanol Selectivity by Nanostructuring Polycrystalline Copper. ChemElectroChem 3, 1012-1019 (2016).

22. Lee, S. H. et al. Oxidation State and Surface Reconstruction of $\mathrm{Cu}$ under $\mathrm{CO}_{2}$ Reduction Conditions from In Situ X-ray Characterization. J. Am. Chem. Soc. 143, 588-592 (2021). 
23. Chen, C. S. et al. Stable and selective electrochemical reduction of carbon dioxide to ethylene on copper mesocrystals. Catal. Sci. Technol. 5, 161-168 (2014).

24. Jiang, K. et al. Metal ion cycling of $\mathrm{Cu}$ foil for selective $\mathrm{C}-\mathrm{C}$ coupling in electrochemical $\mathrm{CO} 2$ reduction. Nat. Catal. 1, 111-119 (2018).

25. Grosse, P. et al. Dynamic Changes in the Structure, Chemical State and Catalytic Selectivity of $\mathrm{Cu}$ Nanocubes during $\mathrm{CO}_{2}$ Electroreduction: Size and Support Effects. Angew. Chem. Int. Ed. 57, $6192-6197$.

26. Liu, H. et al. Scalable synthesis of hollow Cu2O nanocubes with unique optical properties via a simple hydrolysis-based approach. J. Mater. Chem. A 1, 302-307 (2012).

27. Scott, S. B. et al. Absence of Oxidized Phases in Cu under CO Reduction Conditions. ACS Energy Lett. 4, 803-804 (2019).

28. Lum, Y. \& Ager, J. W. Stability of Residual Oxides in Oxide-Derived Copper Catalysts for Electrochemical CO2 Reduction Investigated with 18 O Labeling. Angew. Chem. Int. Ed Engl. 57, $551-554$ (2018).

29. Eilert, A. et al. Subsurface Oxygen in Oxide-Derived Copper Electrocatalysts for Carbon Dioxide Reduction. J. Phys. Chem. Lett. 8, 285-290 (2017).

30. De Luna, P. et al. Catalyst electro-redeposition controls morphology and oxidation state for selective carbon dioxide reduction. Nat. Catal. 1, 103-110 (2018).

31. Löffler, M., Mayrhofer, K. J. J. \& Katsounaros, I. Oxide Reduction Precedes Carbon Dioxide Reduction on Oxide-Derived Copper Electrodes. J. Phys. Chem. C 125, 1833-1838 (2021).

32. Kwak, J. et al. Oxidation behavior of graphene-coated copper at intrinsic graphene defects of different origins. Nat. Commun. 8, 1549 (2017).

33. Arán-Ais, R. M., Scholten, F., Kunze, S., Rizo, R. \& Roldan Cuenya, B. The role of in situ generated morphological motifs and $\mathrm{Cu}(\mathrm{i})$ species in $\mathrm{C} 2+$ product selectivity during $\mathrm{CO} 2$ pulsed electroreduction. Nat. Energy 5, 317-325 (2020). 
34. Grumelli, D., Wurster, B., Stepanow, S. \& Kern, K. Bio-inspired nanocatalysts for the oxygen reduction reaction. Nat. Commun. 4, 1-6 (2013).

35. Andersson, K. et al. Bridging the Pressure Gap in Water and Hydroxyl Chemistry on Metal Surfaces: The Cu(110) Case. J. Phys. Chem. C 111, 14493-14499 (2007).

36. Li, Z. et al. Effect of airborne contaminants on the wettability of supported graphene and graphite. Nat. Mater. 12, 925-931 (2013).

37. Kidambi, P. R. et al. Observing Graphene Grow: Catalyst-Graphene Interactions during Scalable Graphene Growth on Polycrystalline Copper. Nano Lett. 13, 4769-4778 (2013).

38. Álvarez-Fraga, L. et al. Oxidation Mechanisms of Copper under Graphene: The Role of Oxygen Encapsulation. Chem. Mater. 29, 3257-3264 (2017).

39. Biesinger, M. C. Advanced analysis of copper X-ray photoelectron spectra. Surf. Interface Anal. 49, 1325-1334 (2017).

40. Deng, Y., Handoko, A. D., Du, Y., Xi, S. \& Yeo, B. S. In Situ Raman Spectroscopy of Copper and Copper Oxide Surfaces during Electrochemical Oxygen Evolution Reaction: Identification of CullI Oxides as Catalytically Active Species. ACS Catal. 6, 2473-2481 (2016).

41. Yin, X. et al. Evolution of the Raman spectrum of graphene grown on copper upon oxidation of the substrate. Nano Res. 7, 1613-1622 (2014).

42. Yang, J., Wan, X., Tie, S., Lan, S. \& Gao, X. Crystal-facet-controllable synthesis of Cu2O micron crystals by one-step, surfactant- and capping agent-free method and the formation mechanism. Solid State Sci. 104, 106203 (2020).

43. Wang, Y. et al. Catalyst synthesis under $\mathrm{CO} 2$ electroreduction favours faceting and promotes renewable fuels electrosynthesis. Nat. Catal. 3, 98-106 (2020).

44. Eilert, A., Roberts, F. S., Friebel, D. \& Nilsson, A. Formation of Copper Catalysts for $\mathrm{CO}_{2}$ Reduction with High Ethylene/Methane Product Ratio Investigated with In Situ X-ray Absorption Spectroscopy. J. Phys. Chem. Lett. 7, 1466-1470 (2016). 
45. Speck, F. D. \& Cherevko, S. Electrochemical copper dissolution: A benchmark for stable CO2 reduction on copper electrocatalysts. Electrochem. Commun. 115, 106739 (2020).

46. Arán-Ais, R. M. et al. Imaging electrochemically synthesized $\mathrm{Cu} 2 \mathrm{O}$ cubes and their morphological evolution under conditions relevant to $\mathrm{CO} 2$ electroreduction. Nat. Commun. 11, $3489(2020)$.

47. Huang, W.-C., Lyu, L.-M., Yang, Y.-C. \& Huang, M. H. Synthesis of Cu2O nanocrystals from cubic to rhombic dodecahedral structures and their comparative photocatalytic activity. J. Am. Chem. Soc. 134, 1261-1267 (2012).

48. Pérez Sánchez, M. et al. A mechanistic approach to the electroformation of anodic layers on copper and their electroreduction in aqueous solutions containing $\mathrm{NaHCO} 3$ and $\mathrm{Na} 2 \mathrm{CO} 3$. Electrochimica Acta 38, 703-715 (1993).

49. Tang, W. et al. The importance of surface morphology in controlling the selectivity of polycrystalline copper for $\mathrm{CO} 2$ electroreduction. Phys. Chem. Chem. Phys. 14, 76-81 (2012).

50. Marcus, P., Maurice, V. \& Strehblow, H.-H. Localized corrosion (pitting): A model of passivity breakdown including the role of the oxide layer nanostructure. Corros. Sci. 50, 2698-2704 (2008).

\section{Acknowledgements}

This project has received funding from the European Union's Horizon 2020 research and innovation programme under grant agreement No. 732840-A-LEAF.

\section{Author contributions}

K.B., T.-H.P., and M.L. conceived and designed the experiments. R.G. and M.L. supervised the project and led the collaboration efforts. K.B., T.-H.P., and P.A. carried out experiments and obtained the data. The experimental data was analyzed by K.B., F.P.C., R.G., Y.L., and M.L. and discussed by all the authors. The manuscript was written by K.B. and M.L. with contributions from all the authors.

\section{Competing interests}

The authors declare no competing financial interests.

\section{Additional information}

